\title{
Outpatient Cataract Surgery 1982-1986
}

\author{
P. D. DAVIES, E. LIMACHER and K. POWELL \\ Norwich
}

In the United States of America, over the past five or six years, there has been a major change in the way cataract surgery is performed. In 1980 approximately 85 per cent of all cataract surgery was undertaken on hospital inpatients but by 1986 the reverse was true and over $80 \%$ of all patients undergoing cataract surgery were estimated to have had their operation as an outpatient. ${ }^{\prime}$ A similar percentage of cataract surgery is undertaken on an outpatient basis in Stockholm, Sweden but in general, Europe has adopted a much more conservative approach and most operations are still performed on hospital inpatients. In 1978, impressed by the work of Galin and his co-workers, ${ }^{2}$ we felt that the operation for cataract had become such an intrinsically reliable procedure that surgery could be performed safely on outpatients. We began using the technique on selected patients and as we gained experience, confirmed that this was a clinically safe procedure. We therefore sought to extend the availability of outpatient cataract surgery to routine Health Service patients and in 1982 this was made possible by means of a grant from the Department of Health and Social Security who at that time was offering financial support for the introduction of innovative methods of health care. At that time only Ingram ${ }^{3}$ and his team at Kettering were performing outpatient cataract surgery on a systematic basis in the United Kingdom.

Most of the cataract surgery performed in the United States is in Ambulatory Surgical Centres (ASC) and is undertaken on what would be termed private patients in the United Kingdom, under the close and continuing care of one particular surgeon. In the National Health Service, conditions are very different with a heavy reliance on junior doc-

tors and nursing staff, many of whom are training with the inevitable consequence that it is more difficult both to ensure and to monitor the quality of care. In this report we give the results of our experience with outpatient cataract surgery in the Norwich Health District over the five years from 1982 to 1986.

It is important to realise that it was not economic necessity which led us to begin outpatient cataract surgery in Norwich, but rather that as a result of technical advances, cataract extraction has become such an intrinsically safe procedure that it can be safely undertaken on outpatients so avoiding the disadvantages of a hospital admission and yet maintain the excellent results that are now achieved with conventional inpatient surgery. There are, however, considerable financial benefits and in Norwich where there was a waiting list of some three years in the presence of some excess operating theatre capacity, it did offer us the possibility of increasing our surgical through-put without the high initial and subsequent running costs of establishing additional beds.

\section{The Unit}

When we began systematic outpatient surgery, we decided to attempt to adhere to three rules:

(i) We would not use hospital transport

(ii) We would not use hospital beds

(iii) We would arrange for the patients to be seen for the first post-operative visit in their own home.

The attraction for patients of being able to have their surgery within a maximum of eight to ten weeks from their visit to the Outpatient Department has proved considerable and it has surprised us how effectively they have 
been able to arrange transport to and from the hospital on the day of their operation, either by family, friends or even taxi. Hospital transport has been required in a few exceptional circumstances, but this has amounted to less than $1 \%$ of our cases, despite the Norwich Health district being one of the largest in the United Kingdom.

We do not feel that in the absence of general anaesthesia, it is necessary for a patient to lie flat in bed following cataract surgery, particularly as wound closure is now so reliable. Indeed we felt it would be psychologically important for patients not to go to bed either before or after their operation if they were to be mobilised and returned home within a few hours. At no time do our patients therefore use hospital beds but we do have a sitting room reserved for their use where comfortable armchairs are provided. The patients bring their own clean night clothes and dressing gowns with them but if this is not possible, then hospital garments are provided.

In view of the size of our Health District with out patients living up to 30 miles from the Eye Unit, we felt that we would follow initially the practice of Ingram and Banerjee in their Unit at Kettering and arrange for trained ophthalmic nurses to visit the patients in their own home on the first post-operative day. This has in general worked extremely well but there are strong arguments for the patient to return to the hospital at least for their first post-operative visit before being fed into the normal outpatient clinic for their subsequent follow-up. In this study, 767 patients had outpatient cataract surgery and of these 592 were seen for their first post-operative visit in their own home by an ophthalmic nurse and the remaining 175 returned to hospital for examination on the day after operation.

Our management of a typical patient is as follows:

(i) Patients are listed for cataract extraction, either as an outpatient or inpatient at the discretion of the Consultant or Senior Registrar in the course of a normal outpatient clinic attendance. It is discussed with the patient at this time whether they would prefer inpatient or outpatient surgery and the advantages and disadvantages of each method is explained with any particular indications being brought into the discussion as necessary. If, for example, a patient has poor general health which would make general anaesthesia unwise, this is made clear.

(ii) About one month before their projected date of operation, the patient attends a special outpatient clinic with their relative or friend who will be responsible for inserting their post-operative drops. Here they meet the nurses who will be looking after them throughout the day of their operation and who will see them subsequently in their own home. The patients are assessed clinically by the Senior House Officer attached to the Day Case Unit. There are two ophthalmic trained staff nurses attached to the Day Case Unit, one full time and one part time, and they discuss with the patient the programme for their surgery and also perform an A-Scan so that if an intra-ocular implant of a power outside the stock range is required, this can be specifically ordered. The precise date of surgery is agreed, which enables relatives or friends to make suitable arrangements for transport in plenty of time. They are also instructed in the method of inserting drops safely. If the nurse has any doubt concerning the home environment and the support available, she visits the patient's home before a date for operation is agreed.

(iii) On the day of operation the patient arrives at the hospital between 8.15 and 8.30 am when they are briefly examined by both the Senior House Officer and the nursing staff. No pre-operative topical antibiotics are used and for extra-capsular surgery, which is now our routine procedure, pupil dilatation is begun one hour pre-operatively with Cyclopentolate $1 \%$ and Phenylephrine $10 \%$.

(iv) Patients are encouraged to walk to and from the operating theatre which adjoins the ward. Initially we used conventional trolleys but as time passed 
there seemed no advantage in such an arrangement, requiring as it does the use of a theatre porter. Surgery begins at 9.15 am and three or four cases are performed during each operating session.

(v) When the study began the routine operation was an intra-capsular cataract extraction via a corneal section but as will be discussed later, this has changed over the intervening years and we now routinely use extra-capsular surgery utilising the endocapsular technique with a J-loop posterior chamber intraocular implant 'placed in the bag'. Local anaesthesia is effected by a 'cocktail' comprising $5 \mathrm{mls}$ of $0.5 \%$ Bupivacaine together with $5 \mathrm{mls}$ of $2 \%$ Lignocaine mixed in the same $10 \mathrm{ml}$ syringe of which $3 \mathrm{mls}$ are given as a retro-bulbar injection, $1 \mathrm{ml}$ along the superior rectus and the remainder as a facial block. This is followed by orbital compression for 10 minutes by the nurse who has assessed the patients pre-operatively, who has been responsible for their care on the day of operation prior to them coming to the operating theatre and who will remain with the patients throughout their operations. This continuity of care by staff with whom the patients have developed a trusting and confident relationship is in our opinion an extremely important part of successful outpatient surgery. The nurse holds the patient's hand during the operation and this provides a very useful indication to the surgeon if the patient should become unduly anxious or distressed. It is similarly important for the surgeon to be able to chat to the patient during surgery. A subconjunctival injection of Gentamicin 20 mgms together with Depo-Medrone $20 \mathrm{mgms}$ is given at the end of the procedure and the patient returns to the ward for coffee or lunch.

(vi) Each patient is examined at 2 pm by the consultant surgeon who is responsible for their care and they are then free to return home. A simple instruction sheet with a few 'do's and don'ts' is provided and the eye is covered with a pad and
Cartella shield overnight since the local anaesthetic persists for some 18 hours.

(vii) The ophthalmic trained staff nurse who has looked after the patients throughout their treatment, visits the patients in their own home the following morning. She has the ability to perform applanation tonometry if required and if she is worried she may also bring the patient back to hospital in her hospital car for a medical examination. The usual postoperative treatment consists of Cyclopentolate $1 \%$ drops daily together with Maxitrol drops t.d.s. and the patient returns for a further outpatient visit on the fourth post-operative day.

\section{Results}

The total number of patients operated on over the five year period 1982-1986, is shown in Table I together with the type of surgical technique used. All patients who underwent an intra-capsular lens extraction in whom an IOL was inserted had a flexible anterior chamber lens, either a Cilco Multiflex, Iolab 85JM or Pharmacia 350B. Where an IOL was inserted in a patient having an extra-capsular lens extraction this was either a Cilco PC23LRU, Iolab $706 \mathrm{G}$ or Pharmacia 500A. The Table illustrates the change in our surgical technique from predominantly intra-capsular lens extractions in 1982 to an extra-capsular technique in 1986 . This conversion to extra-capsular surgery has taken place rather later in our Unit than many other units, mainly because of the excellent results obtained with intra-capsular surgery and it was only the development of the endocapsular technique with in the bag' placement of a posterior chamber intra ocular lens (IOL) that encouraged us to change.

In 1982, 119 patients had outpatient cataract surgery and of these $63(53 \%)$ received an IOL. In addition there were 7 cases who received an anterior chamber IOL as a secondary procedure. One hundred and three patients $(87 \%)$ had an intra-capsular lens extraction and in only 16 cases was an extracapsular technique used. Five years later in 1986 the change in our surgical technique meant that of the 136 patients who underwent 
Table I. Outpatient cataract surgery 1982-1986

\begin{tabular}{lcccccc}
\hline & ICLE & ICLE/IOL & ECLE & ECLE/IOL & $2^{\circ} I O L$ & Total \\
\hline 1982 & 44 & 59 & 12 & 4 & 7 & 126 \\
1983 & 52 & 93 & 9 & 16 & 6 & 176 \\
1984 & 43 & 94 & 10 & 15 & 9 & 171 \\
1985 & 17 & 61 & 12 & 106 & 9 & 148 \\
1986 & 4 & 13 & 13 & 190 & 39 & 74 \\
\hline Total & 160 & 320 & 56 & & & 765 \\
\hline
\end{tabular}

ICLE = Intracapsular Lens Extraction

ICLE/IOL = Intracapsular Lens Extraction + Intraocular Lens

ECLE $=$ Extracapsular Lens Extraction

ECLE/IOL = Extracapsular Lens Extraction + Intraocular Lens

$2^{\circ} \mathrm{IOL}=$ Secondary Intraocular Lens

primary cataract extraction on an out-patient basis, $119(87 \%)$ had an extra-capsular extraction. The proportion of patients who had an IOL had also risen to $87 \%$.

Initially we selected uncomplicated cases for outpatient cataract surgery, excluding those patients with co-existent glaucoma, whether medically or surgically controlled, those requiring combined glaucoma and cataract surgery, diabetics, high myopes and those with a history of previous uveitis. As our experience has accumulated and with the adoption of extra-capsular surgery, we have eased these restrictions and now operate on cases of surgically controlled glaucoma, those requiring combined glaucoma and cataract surgery, high myopes and those diabetics who have stable control. We still prefer to admit, at least over-night, those who have a history of recent uveitis and also those with unstable diabetes.

The surgical technique used for outpatient surgery is no different from that used on our inpatients. In this study one surgeon (PDD) performed all the surgery and since we undertake one outpatient operating list and one inpatient operating list on successive days each week, it has been possible to obtain a clear comparison between the two groups. We have been primarily concerned with the question of whether outpatient cataract surgery carries with it complications as a result of the patient's early discharge and if so, the incidence of such complications. Over the period of this study a total of 668 patients had a cataract on an inpatient basis compared with the 767 as outpatients. The number of out-

patients who required conversion to inpatients was therefore felt to be an accurate indication of the problems which may have been more appropriately cared for on an inpatient basis. The majority of these were not complications specifically due to their management as outpatients but rather the result of cataract surgery in general.

Retrobulbar haemorrhage is a direct result of local anaesthesia, or, more particularly, the retrobulbar injection, and occurred in 6 cases $(0.8 \%)$. One of these was sufficiently severe to warrant admission, as we felt there was a risk that corneal exposure might occur, although in fact this fear proved unfounded. The remainder were discharged and of these 5 patients, 4 later underwent cataract extraction as outpatients, again under local anaesthesia, without complication some 4 weeks later. The remaining case elected to have surgery under a general anaesthetic as an inpatient. The other complications in outpatients that required admission are listed in Table II and it will be seen that in addition to the case of retrobulbar haemorrhage, there were 3 others who required retention in hospital on the day of their cataract extraction. One of these had a

Table II. Outpatients requiring admission

4 cases on day of operation

4 cases admitted subsequently
1 retrobulbar haemorrhage 1 vitreous loss

1 hyphaema

1 nausea

Day 1. Raised IOP

Day 2. Raised IOP

Day 3. Hyphaema

Resuturing of incision 
large vitreous loss and one an hyphaema of more than $50 \%$ noted on examination before the patient returned home. The remaining case was a patient who claimed to have severe nausea so that she felt unable to face the journey home by car, although in every respect her ocular condition was unexceptional and in particular her intra-ocular pressure was normal.

Four other cases were admitted between one and three days following outpatient cataract surgery. Two cases of secondary glaucoma with corneal oedema were admitted, both following intra-capsular surgery, one case 24 hours after operation and the other 48 hours. Two other patients were admitted on the third day following operation, one of whom had a total hyphaema and the other a broken corneal suture and leaking wound. The total number of patients admitted therefore was 8 $(1 \%)$ of the total 767 who underwent outpatient cataract surgery. All the patients who required admission followed intra-capsular lens extraction and to date no patient has been admitted following extra-capsular lens extraction.

The other major complication resulted from the use of anterior chamber IOLs. Two of these needed repositioning under local anaesthesia on an outpatient basis when one or more feet of the implant were found to be in the posterior lip of the section. Five IOLs were also removed as a result of excessive mobility and consequent displacement. Four of these were replaced with alternative implants whilst one was not replaced as damage occurred to the iris during its removal. It was thought that incorrect initial IOL selection was the reason for the poor fit of the five implants that required removal.

\section{Discussion}

In the United Kingdom, general anaesthesia is readily available and of a high quality. Nevertheless, in the elderly population, most surgeons are familiar with the post-operative complications that are directly attributable to the anaesthetic, rather than to the surgery. In the Norwich Health District, $87 \%$ of our patients presenting for cataract extraction are over the age of 60 years and $82 \%$ over 70 years of age. Whilst these figures will vary somewhat between Health Districts, they can be considered generally representative of the United Kingdom. Despite the routine use of anti-emetics, post-operative vomiting remains a significant problem after general anaesthetic occurring in $6 \%$ of our cataract patients. As a result, many patients are reluctant to return home as soon as their ocular condition allows. In addition $15 \%$ of our inpatients required 7 or more days in a convalescent home before they were able to return to their own homes. Similarly, $60 \%$ of our inpatients required hospital transport in order to return home, whereas this proved necessary in less than $1 \%$ of our outpatients undergoing cataract surgery.

In this Unit, we routinely present our patients with a questionnaire and this has revealed that $20 \%$ of patients in whom cataract extraction is indicated have a resistance to hospitalisation and are relieved to be offered the prospect of their surgery on an outpatient basis. All those patients who underwent outpatient cataract surgery were questioned six months after the procedure about their attitude to the experience. Eighty-four per cent of those having their first cataract extraction on an outpatient basis preferred to have their second operation in the same manner but of those patients who had one eye operated on under general anaesthesia as an inpatient and one eye on an outpatient basis under local anaesthesia, $92 \%$ preferred the latter.

Outpatient surgery does, however, carry certain disadvantages. Approximately $15 \%$ of patients dislike the prospect of their surgery under a local anaesthetic although a sympathetic and confident explanation by the surgeon does alleviate this anxiety in many cases. There are also a number of patients for whom, for clinical reasons, it is felt that a general anaesthetic is preferable. There is no doubt that outpatient surgery must be of a high quality to be safe and that an experienced surgeon is therefore necessary. Many surgeons find the operation more stressful under local anaesthetic and the desirability of chatting to the patient during surgery in order to maintain their sense of confidence is an additional distraction they would prefer to avoid. A high degree of discipline and organisation is 
also necessary within the operating theatre amongst all the staff, since a thoughtless word can generate a great deal of anxiety in the patient's mind. Certainly it is not a procedure which lends itself to the training of junior staff and this is a major disadvantage within the National Health Service.

The economic advantages of outpatient cataract surgery have been calculated in the Norwich Health District at $45.19 \%$ of the cost of the average inpatient admission for three nights plus general anaesthesia. An additional reduction of approximately $4 \%$ would be gained if the home visit on the first post-operative day was replaced by an assessment in the hospital. This is the procedure now in use at Norwich and thus the cost of outpatient cataract surgery is reduced to approximately $41 \%$ of an inpatient case.

The standard surgical procedure currently used for both inpatient and outpatient cataract surgery, whether under general or local anaesthesia, is an extracapsular cataract extraction using an endocapsular technique with a posterior chamber modified J-loop intra-ocular implant placed in the capsular bag. The cost of such a procedure under general anaesthesia in the Norwich Health District where the average hospital stay is three nights has been calculated at $£ 807.87$. The element for transport and convalescence is calculated on the basis of $60 \%$ of inpatients in Norwich requiring hospital transport to return home on discharge at an average journey cost of $£ 10.40$, therefore $60 \% \times £ 10.40=$ $£ 6.24$ has been added to each patient's costs.

Similarly $15 \%$ of our inpatients go to convalescent homes for an average of 10 days at a daily cost of $£ 46.00$. Thus $15 \% \times £ 460=$ $£ 69.00$ has been added to the costs of each inpatient.

The total figure for an inpatient cataract extraction has been calculated as follows:

\section{Salaries and wages}

Consultant Ophthalmologist

Consultant Anaesthetist

S.H.O.

4 Nurses

O.D.A.

O.D.O.

Recovery Nurse
Disposables, including intra-ocular

implant, drugs, dressings,

medical gases etc.

313.92

Transport and convalescence

In patient stay

Average inpatient cost per day

$\times 3$ nights

364.00

Grand Total $\overline{£ 805.87}$

The cost of the same procedure performed on an out-patient basis under local anaesthesia totals $£ 364.21$ and is calculated as follows:

\section{Salaries and wages}

Consultant Ophthalmologist

$£ 13.50$

S.H.O.

4 Nurses

15.48

Disposables, including intra-ocular

implant, drugs, dressings, etc.

287.49

Day Case stay

44.26

\section{Grand Total $\overline{£ 364.21}$}

No allowance has been made for the depreciation of surgical instruments since depreciation accounting is not undertaken at all in the National Health Service.

It is interesting how small a proportion of the total cost is that of the salaries for the highly skilled staff involved. The comparison of the staffing levels and facilities required for cataract surgery as an inpatient and outpatient respectively is summarised in Table III.

\section{Conclusions}

The high level of patient acceptance of outpatient cataract surgery together with the low level of complications attributable to surgery on this basis has led us to conclude that, in the Norwich Health District, $80 \%$ of all cataract surgery could be performed in this way. The avoidance of the complications of general anaesthesia and hospitalisation in the elderly are an additional attraction whilst the considerable financial savings confirm our opinion that, in theory, this is the method of choice for most cataract surgery. When our commitment to training of medical and nursing staff within the National Health Service is taken into consideration and also those patients in whom general anaesthesia is clinically neces- 
Table III. Comparison of staff and facilities required for cataract surgery

\begin{tabular}{lll}
\hline \multicolumn{1}{c}{ Inpatient } & & \multicolumn{1}{c}{ Outpatient } \\
\hline 3 nights & Admission & $\begin{array}{l}6 \text { hours } \\
\text { (no use of hospital beds) }\end{array}$ \\
$\begin{array}{ll}\text { GENERAL } \\
\text { Theatre Porter }\end{array}$ & Anaesthetic & $\begin{array}{l}\text { LOCAL } \\
\text { Onaesthetist }\end{array}$ \\
$\begin{array}{l}\text { Operating Dept Assistant (ODA) } \\
\text { Recovery nurse } \\
\text { Three theatre nurses }\end{array}$ & & \\
$15 \%$ patients & Convalescence & Three theatre nurses \\
$65 \%$ patients & Ambulance to home & Nil \\
\hline
\end{tabular}

sary for one reason or another, a more realistic estimate of the proportion of patients suitable for outpatient cataract surgery in the average unit, is probably of the order of $40-45 \%$ although variations in this figure will occur depending on local circumstances.

Outpatient cataract surgery is not a means of increasing the surgical through-put of a unit unless excess operating theatre capacity exists relative to the beds available in that unit. It is, as we have shown, a means of considerably reducing the cost of a cataract operation but above all, it is our opinion that current surgical techniques make it unnecessary for the majority of patients to stay in hospital, even over night for routine cataract surgery.
We would like to thank the Medical and Nursing Staff of the Department of Ophthalmology, West Norwich Hospital, for their assistance and encouragement in the establishment of routine Outpatient Surgery. We are grateful to the Finance Department of the Norwich Health District for their assistance in determining the relevant costs of outpatient and inpatient surgery and finally to Mr Geoffrey Bluckert, Unit Administrator, West Norwich Hospital when the study was begun, whose support proved invaluable.

\section{References}

'Severin, SL: Out-Patient Cataract Surgery. Seminars in Ophthalmology, 1986; 1: 130-5.

${ }^{2}$ Galin, MA, Bowiuk, MA, Obstbaum, S: Hospitalisation and Cataract Surgery. Ann Ophthalmol, 1981; 13: 365-7.

${ }^{3}$ Ingram, RM, Bannerjee, D, et al.: Day Case Cataract Surgery. Trans Ophthalmol Soc UK 1980, 100: $205-9$. 